\title{
Alteración del rol sexual en niños
}

\author{
Dra. Eva Hona R., ${ }^{1}$ Srta. Ana María Morales D, , Srta, Paulina Torres O. $^{2}$
}

Disturbed gender identity in children

\begin{abstract}
The main features of ten boys with cross-gender aiterations are studied in the present puper. As deseribed in the literature, they showed marked preference for femenine attitudes ard games, rejection of boys games and sports, alfected language and, surne of them, transvestism.

It is worthy to remark three aspects which are not described in the consulted literature: the injportance of femenine figures besides the mother (grand-mother, annts) who lived with the family. A special pattem of personality characterized by shyness, withdrawal, and sornetirnes, fobic tears. Finally, a somatic appearance frecuently characterized by fine feahires sund a delicate and slender resemblance.

It is emphasized that, the sooner the diagnosis is made and the treatunent starts. the better is the prognosis regarding the evolution of their adult sexulity.
\end{abstract}

Durante el último tiempo nos ha toxado atender varios casos'de niños que presentaban alteración de la identidad y rol sexual. Este hecho, conjuntamente con lo exiguo de la literatura al respecto en nuestro medio, nos indujo a estudiar y reunir

\footnotetext{
I Psiquiatra

2Psicólopra

Servicin de Neuropsiquiatrfa Infanti-Adolescente, Area Oxcidente, Hospital Surn Juan de Dios.
}

los distintos casos que consultaban por este motivo. Por otra parte, la bibliografía extranjera existente es bastante reciente, generalmente de la última década. Ello obedece al hecho que anteriormente se tendia a minimizar y restar importancia a este problema y con frecuencia, la respuesta del médico frente a la consulta de los padres era típicamente que "esto va a pasar con el tiempo". Tampoco se había establecido un nexo entre estos problemas de alteración del rol sexual 
en el niño y los problemas de la sexualidad adulta.

Normalmente el desarrollo de la bififación sexual se produce precozmente en el niño. Los años del período preescolar son decisivos para el desarrollo de su personalidad y durante esta etapa se establecen y/o modifican muchas características de gran importancia, entre ellas la tipificación sexual.

Desde el punto de vista psicológico, la tipificación sexual está estrechamente vinculada y es consecuencia de los procesos de identiticación del niño. Mediante la identificación el niño va incorporando y absorbiendo las pautas de conducta del modelo, sus atributos, características y motivos personales y es así un proceso fundamenta] en la socialización del niño.

La tipificación sexual o adquisición del rol sexual es la adopción de conductas, valores, características e intereses propios del sexo del niño. ${ }^{y} \mathrm{En}$ ello intervienen factores tanto personales como la influencia de su ambiente inmediato, especialmente la relación del niño con sus padres y también ciertos patrones y pautas socioculturales. Todo ello influye para que el niño tome como modelo al padre y la niña a la madre.

\section{MATEHLL Y METODO}

Todos nuestros pacientes ${ }^{10}$ fueron remitidos al Servicio de Neurosipquiatría Infantil-Adolescente del Area Occidente, en los años 79 y 80 , con excepción de dos casos, uno del año 72 y otro del año 76, los que en esa oportunidad consultaron por mohivos ajenos a la conducta afeminada Los 10 pacientes son de sexo masculino; no hemos atendido niñas que consulten por problemas de identidad sexual.

Los datos fueron obtenidos en entrevista a las madres en todos los casos; sólo en 3 casos se logró entrevistar a los padres. Los niños fieron entrevistados por 2 de nosotras (un médico y una psicóloga) la mayoría de las veces; algunos casos por uno solo de los profesionales. Todos los niños fieron sometidos a un examen psicométrico para medir su nivel intelectual (test de Wechsler infantil) y al test de Machover (dibujo de la figura humana).

\section{RESULTADOS (Tabla 1)}

A) Edad de consulta: Fluctúa entre los 6 años y los 13 años y medio, observándose que la mayoría $(50 \%)$ consultó entre los 9 y 10 años, aunque la edad de comienzo de los sintomas a conducta desviada precedía por tiempo considerable a la fecha de consulta. La edad de comienzo no se pudo precisar, aunque en la mayoria de nuestros casos ocuría en la etapa preescolar, alrededor de $\operatorname{los} 4-5$ años.

B) Motiuo de consulta: En 5 de nuestros 10 casos, e] motivo principal de consulta fiue por actitudes y

Tabla 1

1ABLA I PRINCIFALES SINTOMAS DBSEKRADOS ENNIIOS WON CONDUCTA AFEMINADA

\begin{tabular}{|c|c|c|c|c|c|c|c|c|}
\hline $\cos 0 \mathrm{M}$ & $\begin{array}{l}\text { Edod se } \\
\text { 19 m mat }\end{array}$ & $\begin{array}{l}\text { Rechaza of } \\
\text { paysos mes. }\end{array}$ & $\begin{array}{l}\text { Preferencio } \\
x \mu \text { enos iem }\end{array}$ & $\begin{array}{l}\text { Act y gesics } \\
\text { 12meninos }\end{array}$ & $\begin{array}{c}\text { Tronswes } \\
\text { IIsmo }\end{array}$ & $\begin{array}{l}A^{+} \operatorname{cocn} 0 \\
\text { moranllars. }\end{array}$ & $\begin{array}{l}\text { Desea de } \\
\text { ser mujer }\end{array}$ & $\begin{array}{l}\text { Dbup fig } \\
\text { 'emenino }\end{array}$ \\
\hline$?$ & $12 \mathrm{~s} 6 \mathrm{~m}$ & + & \pm & + & + & - & $n t$ & - \\
\hline 2 & $1306 \mathrm{~m}$ & + & + & + & $\cdots$ & - & $\rightarrow$ & $=$ \\
\hline 3 & 100 & + & + & + & + & - & $-12)$ & + \\
\hline 4 & $9 c 9 m$ & \pm & + & + & - & - & - & - \\
\hline 5 & 5o Em & + & + & + & - & - & - & + \\
\hline 6 & $92 \mathrm{6m}$ & \pm & + & - & + & - & + & t \\
\hline 7 & $3 a \quad 6 m$. & + & \pm & + & + & - & - & - \\
\hline 8 & $3 \mathrm{~m}$ & + & + & + & - & - & + & + \\
\hline 9 & 60 & \pm & + & + & - & - & n & + \\
\hline 10 & $7 a$ & + & + & + & + & + & + & + \\
\hline
\end{tabular}


modales afeminados; preferencia por juegos y actividades propias de niñitas. En $\mathbf{4}$ casos se observó el uso de atuendos femeninos que generalmente pertenecían a la madre.

En los casos restantes, el motivo de consulta abarcaba diversos trastomos conductuales, especialmente timidez, temores föbicos y aprehensión; inquietud, irritabilidad, actitud de oposición, etc. y también, problemas de aprendizaje.

C) Intereses y juegos. Relaciones con panes: Todos estos niños rechazan los juegos masculinos por considerarlos bruscos y sentirlos como peligrosos para su integridad personal. Por ejemplo, un niño expresaba "no me gustan porque me pueden llegar pelotazos y patadas y me empujan". Generalmente estos niños prefieren jugar con niñas o solos. Rechazan por ejemplo el fútbol y deportes en general, demostrando preferencia por juegos de tipo sedentario, como juegos de salón, visitas, papá y mamá, a veces con muñecas, imitación de artistas de la TV, etc., "son más entretenidos".

Todos los niños presentaban dificultades para integrarse a grupos de su misma edad y sexo y, generalmente eran a su vez rechazados por los otros niños quienes los tildaban de "mariquita", "cola" y otros apodos peyorativos. En uno de nuestros casos se observó además una exacerbación de intereses, curiosidad y juegos sexuales (observaba a la hermana cuando ésta iba al baño, tocaba el cuerpo a niñas y nin̄os, jugaba con munecas tipo Barbie porque le gustaba "su cinturita", etc.).

Como se señaló, 4 de estos niños se vestían repetidas veces con atuendos femeninos, adoptando en sus juegos el rol de mamá o ce alguna artista. Otro niño se ponía el sostén de la mamá y quería pintarse ojos y boca cuando ella se maquillaba. Tres de nuestros niños manifestaron abiertamente su deseo de haber sido niñita; en otro caso, si bien el niño nunca expresó que lo hubiese deseado, nos cabe la duda respecto a la veracidad de su respuesta y nos parece que hubo ocultamiento $y$ evasión.

D) Apariencia extema: En 6 de estos 10 niños se observa una contextura delgada, aspecto grácil y rasgos finos; voz suave, atiplada, modo de hablar pueril; algunos de ellos con gestos amanerados y modales afeminados. En 3 de los 10 casos se observó un buen desarrollo somático y ausencia de características afeminadas. Uno de los niños era obeso, de formas redondeadas; en este caso y en otros 2 existía el antecedente de una criptorquidia.

E) Descripción de personalidad y nivel intelectual: La mayoria ${ }^{6}$ de nuestros casos presentaba un nivel intelectual normal; 3 niños tenían un nivel intelectual normal lento y uno era un niño limítrofe de base orgánica.

En cuanto a características de personalidad, en una amplia mayoría ( $80 \%$ ) se observó predominio de rasgos tales como: timidez, inseguridad, introversión y dificultad en sus relaciones especialmente con coetáneos; ansiedad, temores frecuentemente fóbicos; tendencia a aislarse y retraerse. En 2 de estos 8 niños se observaron conductas de tipo obsesivo, rasgos perfeccionistas, apego al orden y cuidado excesivo de sus pertenencias.

Solamente en 2 de nuestros casos $(20 \%)$ se observaron conductas de tipo extrovertido, tales como tendencia a llamar la atención, cierto histrionismo y desinhibición.

Tres de los 10 niños manifestaron abiertamente que habrían preferido ser mujeres, uno de ellos incluso pedía que lo operaran (cuando tenía 8 años). Otro niño manifestaba rechazo por sus órganos genitales, "me dan asco". Otros al consultárseles sobre ello manifestaban aceptación por su sexo.

En el test de Machover (dibujo de la figura humana) se encontró que el 60\% de los niños dibujó una figura femenina. Uno de los restantes dibujó una figura masculina pero incompleta e infantil, no acorde con su nivel intelectual y edad. Los otros 3 nin̄os dibujaron una figura masculina adecuada, siendo llamativo que uno de ellos acentuara los rasgos sexuales secundarios (bigote, vello en el tórax, carácter deportivo) lo que contrastaba con su propia apariencia obesa $y$ de formas redondeadas. (Figuras 1,2 y 3 ).

F) Otros sintomas: La mayoría de nuestros casos presentaba otros síntomas aparte de las alteraciones del rol sexual ( $80 \%$ ), observándose conductas frecuentes que son motivo de consulta en psiquiatría infantil, tales como inquietud; trastomos de aprendizaje ( 3 disléxicos); enuresis noctuma (1 caso); retraso del lenguaje (l caso) y un niño con una hipoacusia severa. Uno de estos niños habia consultado a los 5 años 6 meses por conductas francamente alejadas de lo normal y que sugerían una pre-psicosis. Solamente $\mathbf{2}$ niños no manifestaban otros síntomas o problemas fuera del problema de alteración del rol sexual. 


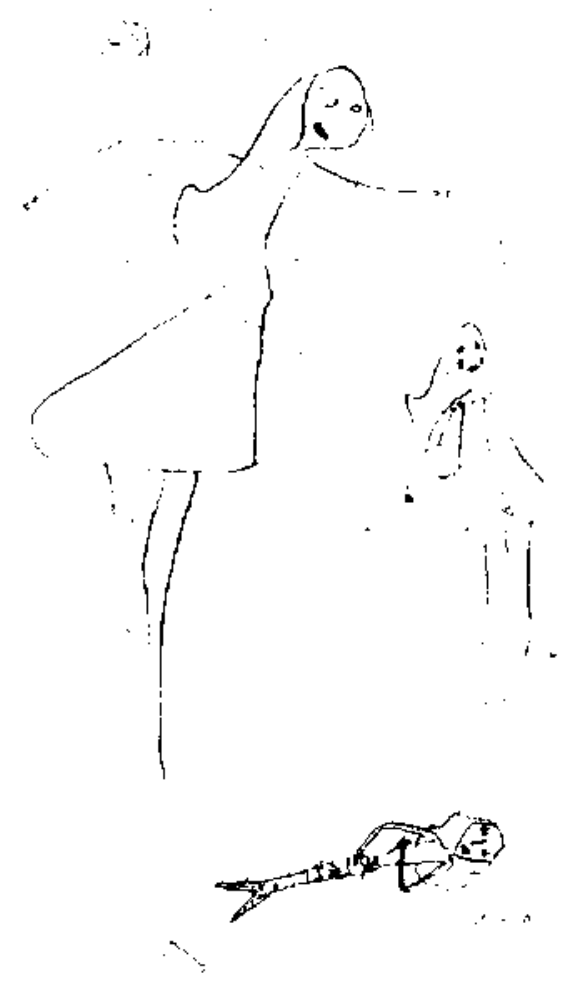

Figura 1

G) Contexto familiar: Analizando el cuadro familiar llama la atención que la mayoría de los niños provenían de hogares mal constituidos, por ausencja de uno de los cónyuges, ya sea por separación o muerte. También hogares inestables y conflictivos por desaveniencias entre los padres. En varios de nuestros casos era significativa la importancia de figuras femeninas tales como abuelas y tias con las que el niño vivía, manteniendo una relación afectiva muy estrecha y frecuentemente dependiente. Por ejemplo, un caso típico que ilustra este hecho es el de R.T., niño que a los 6 años sufrió la pérdida de su madre por fallecimiento. El padre lo llevó a vivir a casa de la abuela paterna en otra ciudad, donde una tía soltera tomó especiál afecto a este niño, mimándolo y consintiéndolo en forma excesiva. Aunque el padre lo visitaba semanalmente, la ligazón afectiva era mucho más fuerte con la tía que con el papá. Cuando éste se casó nuevamente y se trajo a R.T. consigo, observó que el niño presentaba conductas francamente afeminadas que contrastaban con las de su hermano mayor quien no había recibido los desbordes afectivos de dicha tia.

En 2 casos el niño no tenía ningún contacto con el padre, quien había desertado del hogar hacía
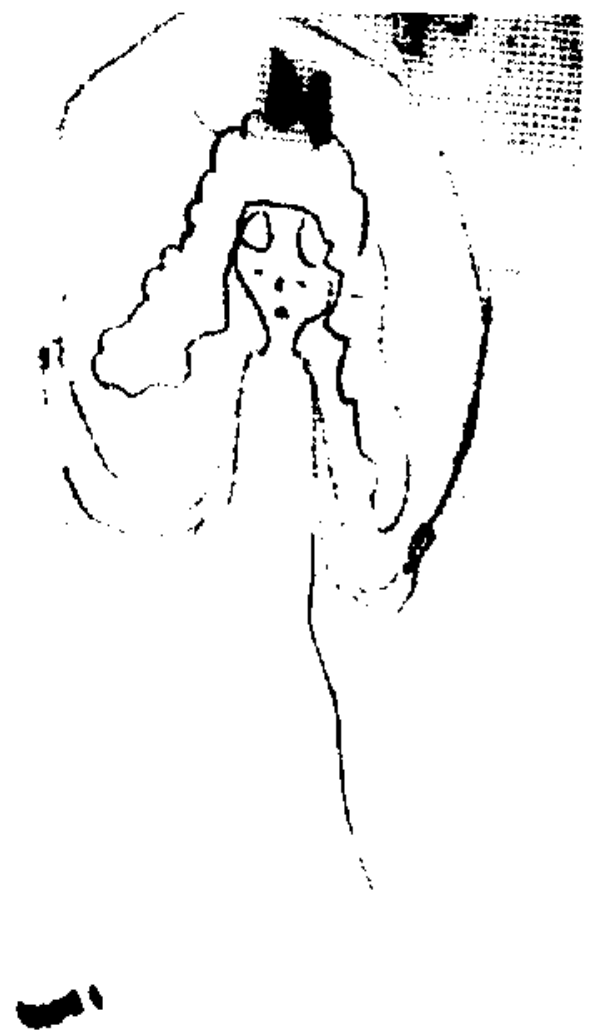

Figura 2

Figura 3

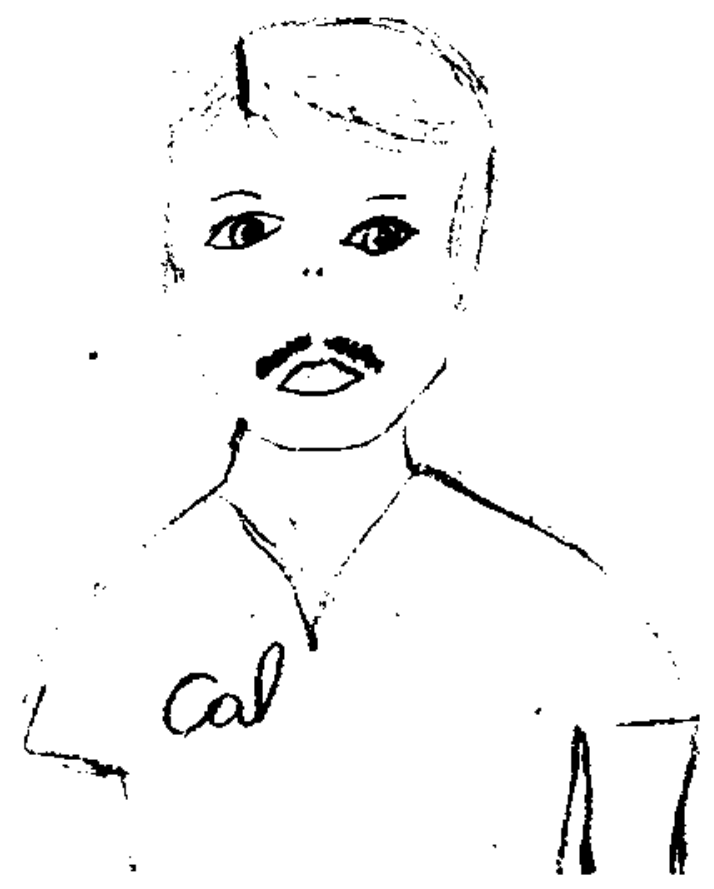


años. En otros casos, a pesar que el padre estaba en el hogar, la relación de éste con el niño era muy superficial y el contacto escaso y de poco valor afectivo. Estos padres se dedicaban a su trabajo, eran responsables en cuanto a la mantención de] hogar, pero rara vez compartian con sus hijos. (Una madre describía esto expresando que "es como si una estuviera sola").

Frecuentemente se observó una relación muy estrecha, a veces dependiente, con una convivencia cerrada e intima entre madre e hijo. Estas madres se caracterizaban por ser casi todas más bien dominantes, firmes, decididas y activas.

Más raro de observar - sólo en 2 casos- fue la mala relación con la madre. En estos casos el niño mantenía un mejor vínculo con el padre, siendo la madre fría, bastante castigadora, restrictiva y con rasgos neuróticos. En uno de nuestros casos (e] nin̄o con rasgos prepsicóticos de base orgánica) el contexto familiar era particularmente alterado: huérfano de padre y madre, a temprana edad quedó a cargo de una tía abuela, siendo confiado a una cuidadora de edad avanzada quien era la única que mantenia un vínculo afectivo significativo $\mathrm{e}$ importante con el niño.

H) Nivel socioeconornico: Siete nin̄os provenian de un ambiente de nivel medio-bajo y 3 niños de un nivel socioeconómico medio. Esta distribución refleja aproximadamente el nivel socioeconómico de los consultantes del sector atendido por el Servicio.

No se observó mayor influencia de este aspecto en el tema que nos interesa.

I) Euolución y Trutamiento: El enfoque terapéutico consistió básicamente en manejo ambiental e indicaciones a los padres o adultos responsables. A grandes rasgos, esto se realizó enfatizando la relación afectiva armónica entre padre e hijo; alentando un mayor y mejor contacto $y$ participación en actividades comunes; fomentando una relación más equilibrada y estable en los roles paterno y materno. Se señaló la importancia que el padre, a través de un cambio de actitud, proveyera al niño de un modelo adecuado de identificación.

Por otra parte, en los casos en los cuales había una participación excesiva y negativa de tías y abuelas en la crianza y formación del niño, se indicaron medidas tendientes a minimizar estas influencias. De los 10 casos, 3 niños recibieron psicoterapia individual conjuntamente con el manejo ambiental.
De nuestros 10 casos, 2 no asistieron más que a 2 sesiones por lo que no podemos afirmar nada sobre su evolución. En 6 casos se observó una respuesta favorable; en 3 de éstos las medidas de manejo ambiental anteriormente descritas fueron suficientes, mientras que los otros 3 requirieron psicoterapia individual. En 2 casos el pronostico es francamente desfavorable: uno de ellos es reticente a la psicoterapia, manteniendo una actitud de oposición pasiva; el otro es el niño con diagnóstico previo de pre-psicosis (a los 5 años y medio) y que volvió a consultar por conductas afeminadas a los 13 años y medio, una edad muy tardía para conseguir buenos resultados, más aún tratándose de un menor con un nivel intelectual limitrofe.

\section{DISCUSION}

Las publicaciones revisadas concuerdan en describir el cuadro de la alteración del rol sexual como un síndrome de comienzo precoz en la infancia, que se caracteriza principalmente por la preferencia del niño en cuanto a ropas, juguetes, actividades y compañía del otro sexo; actitudes, gestos y lenguajes amanerados $y$, en ocasiones, el deseo expreso de pertenecer al sexo opuesto. ${ }^{1.2 .3}$, $5,7,12$

Todos estos niños rechazan los juegos considerados como típicamente masculinos y los deportes, prefiriendo jugar a las visitas, muñecas, juegos de salón, adoptando frecuentemente el rol femenino en ellos. Manifiestan un interés desacostumbrado por la moda, peinados y maquillaje. Se preocupan y fijan en la ropa que usa la madre, sus amigas, etc.; les gusta peinar a la mamá y hermanas y muchos de ellos también manifiestan esta preocupación en su propia vestimenta y arreglo personal. $^{7,} 12$

Esta descripción coincide plenamente con lo observado en nuestra casuística. Hay que hacer notar que estas conductas pueden presentarse en niños normales en forma ocasional, momentánea y aislada. La persistencia, variedad y la presencia de una constelación de conductas afeminadas es la que otorga el sello patológico al síndrome.

La mayoría de los autores señala que las conductas afeminadas se presentan precozmente, alrededor del tercer o cuarto año de vida y muchas madres expresan que, hasta donde pueden recordar, el niño manifestó preferencia por juegos y actividades femeninas desde muy pequeño. 2.3 .12 
Este aspecto también coincide con lo observado en nuestros casos, pues aunque algunas madres no precisaban la edad, concordaban en señalar un comienzo precoz.

Generalmente son las madres las que se preocupan de consultar por este problema, mientras que los padres tienden a minimizar y a no prestar ateción a las conductas inadecuadas del hijo., 3, 12 En cierta medida esto refleja, como se ha visto, la deficiente relación padre-hijo observada en estos casos.

En cuanto a la relación de estos niños con sus padres se ha observado un vínculo afectivo más estrecho y dependiente con la madre y una relación más distante y fría con el padre, tal como se hizo notar en el análisis de este aspecto en nuestros casos.

Otra característica del grupo familiar es que la imagen matena es percibida como más dominante, más fuerte y activa; en cambio, la imagen del padre es vista como más pasiva, más débil y lejana $y$ a veces es descalificada por la madre. ${ }^{13}$ Frecuentemente hay una identificación con la figura femenina -ya sea madre, tía, abuela o hermana mayor- lo que se refleja, entre otras cosas, en el dibujo de la figura humana.

Estudios retrospectivos en homosexuales adultos, describen la relación madre-hijo como "intima, cerrada, seductora y comprometida" y la relación padre-hijo como "distante, ausente o pasiva". ${ }^{12}$ Los homosexuales adultos recordaban a sus madres como posesivas y sobreprotectoras y a los padres como rechazantes y poco comprensivos. En los grupos de control de adultos heterosexuales también se encontraban estas dinámicas familiares, pero en una proporción significativamente menor. ${ }^{12} \mathrm{Si}$ bien es cierto que nosotros no hemos hecho estudios en homosexuales adultos o adolescentes, esta descripción parece reflejar la realidad y se encuentra frecuentemente descrita, tanto en la literatura psicológica como en la literatura universal.

\section{CONCLUSIONES}

Del análisis de nuestra casuística se desprende que en numerosos aspectos hay concordancia con lo descrito en la literatura:

1) Es raro o excepcional que una niña sea traída a consulta por conductas masculinas. Al parecer, este tipo de conductas es menos llamativo y mejor tolerado por la sociedad. Así, nuestra casuística está formada exclusivamente por niños del sexo masculino.

2) Comienzo precoz del cuadro (edad preescolar).

3) Características conductuales prácticamente idénticas (preferencias por actividades, juegos femeninos; rechazo por actividades y juegos masculinos así como de compañeros de juego varones; gestos, actitudes y modales afeminados; en ocasiones, transvestismo, etc.)

4) Identificación con figura femenina, lo que se refleja -entre otras cosas- en el dibujo de la figura humana.

5) Hogares mal constituidos y/o inestables.

6) Relación deficiente $y /$ o conflictiva con el padre.

7) Relación estrecha y dependiente con la madre.

8) En cuanto al tratamiento, concondamos con los autores revisados en que mientras más precozmente se inicie (edad preescolar) hay más posibilidades de éxito terapéutico y también, en que en algunos casos, el manejo ambiental parece ser suficiente para lograr la remisión de las conductas afeminadas.

Por otra parte, de nuestro estudio se desprenden algunos hallazgos que no encontramos descritos en la literatura revisada, si bien, está descrito en una casuística chilena publicada en $1979 .{ }^{13} \mathbf{E n}$ primer término, la importancia de figuras femeninas — aparte de la madre- en el contexto familiar, por ejemplo, presencia de tías, abuelas, hermanas mayores, etc., que influían significativamente en la formación del niño en desmedro de la influencia paterna. Este hecho nos parece atribuible a la conformación de la familia chilena de nivel medio-bajo, la que frecuentemente incluye otros familiares o "allegados" al núcleo familiar, en contraste con la familia anglo-sajona descrita en la literatura, que es de tipo nuclear (padres e bijos exclusivamente).

Otro aspecto observado en nuestra revisión se refiere a los rasgos de personalidad encontrados en estos niños: predominio de caracteristicas tales como timidez, inseguridad, introversión, ansiedad, temores fóbicos y tendencia al retraimiento social, especialmente frente a coetáneos del mismo sexo.

Un tercer punto, que no ha sido destacado en la bibliografía, se refiere al aspecto somático que frecuentemente se observa en estos nin̄os: apa- 
riencia física delicada y grácil; rasgos finos, voz suave y pueril. Llama la atención que en 3 de nuestros casos había antecedentes de criptorquidia, lo que sugeriría que una anomalía orgánica hace al niño más permeable a la influencia de factores ambientales que inciden en su identificación sexual.

Del análisis de nuestros casos podemos concluir que este síndrome se estructura principalmente desde un contexto psicosocial. Que su diagnóstico y tratamiento precoz es de relevancia, pues permitirá prevenir las alteraciones de la sexualidad adulta que parece ser el destino de estos nínos si son dejados a su evolución espontánea.

\section{RESUMEN}

Se estudiaron 10 pacientes de sexo masculino, cuyas edades fluctuaban entre los 6 años y los 13 años, los que fueron remitidos al Servicio de Neuropsiquiatría Infantil-Adolescente del Area Occidente por conducta afeminada o por otras causas, descubriéndose en estos últimos en el curso de la anamnesis este tipo de sintomatologia.

Se hace un análisis de la edad de comienzo de los síntomas, de las características de éstos (rechazo por juegos y compañeros de sexo masculino, preferencia por actividades sedentarias, con niñas o solos, etc.) y de la apariencia extema de estos niños. Se pone hincapié en la descripción de ciertas características de personalidad en estos pa- cientes, así como de su contexto famịliar. Se reseña brevemente su evolución y tratamiento.

Finalmente, en las conclusiones se recalca la importancia del diagnóstico y tratamiento precoz en estos casos, a fin de evitar las alteraciones de la sexualidad adulta, lo que parece altamente probable si se dejan evolucionar a su suerte.

\section{REFERENCIAS}

1 Greene, Rlchard "Effeminacy in prepuberal males. Summary of ill cases and recomendations for case management". Pediatrics 27: 286-291, 1961 .

2 Greene, Rlchord. "Childhood cross-gender identification". J. Mental and Nervous Dis. 147: 500509; 1966.

3 Zuger, Bemard "Effeminate behaviour present in boys from early childhood". J. of Pediatrics 69: 1028-1107; 1966.

4 Baker H., Stoler $R$ " "Can a biological force contribute to gender identity ?". Am. J. of Psychiatry 10A: 1653-1658; 1968.

5 Zuger B., Pdsy T. "Effeninate behaviour present in boys from early childhood. II Comparison with similar symptoms in now-effeminate boys". Pediatrics 44: 375-380; 1969.

6 Ajuniaguern "Manuel de Psychiatrie de l'enfant". Ed. Masson et Cie.; 1971.

7 Lebouts Phil, MD. “Feminine behaviour in boys. Aspects of its outcome". Am. J. of Psychiatry 128: 1283-1289; 1972

8 Bates J., Skibeck W., Smikh K, Bendier. "Intervendion with families of gender disturbed boys". Am. J. of Ortopsych. 45: 150-157; 1975.

9 Musser, Conger, Kagon "Desarrollo de la personalidad en el niño". Ed. Trillas; 1979.

10 Greene Richurd. "Atypical psychosexual development". Child Psychiatry. Ed. M. Rutter and R. Hersov-Blackwell. Scientific Public; 1979.

11 Whittaker, Jomes, "Psicología". Nueva Editorial Interamericana; 1977.

12 Greene, Richard "Disturbances of development". Basic Handbook of child psychiatry Vol. 2. Noshpitz; 1979.

13 Guajardo, Humberta, "Desviación del rol de conducta sexual en niños". Hev. Chil. Ped. Vol 50 (4): 6B-71; 1979. 